\title{
Linear Polarization Properties of Parsec-Scale AGN Jets $^{\dagger}$
}

\author{
Alexander B. Pushkarev 1,2,*(1), Yuri Y. Kovalev 2,3,4 (D), Matthew L. Lister ${ }^{5}$, \\ Tuomas Savolainen ${ }^{4,6,7}$ (D), Margo F. Aller ${ }^{8}$, Hugh D. Aller ${ }^{8}$ and Mary A. Hodge ${ }^{5}$ \\ 1 Crimean Astrophysical Observatory, Crimea, 98409 Nauchny, Russia \\ 2 Astro Space Center of Lebedev Physical Institute, Profsoyuznaya 84/32, 117997 Moscow, Russia; \\ yyk@asc.rssi.ru \\ 3 Moscow Institute of Physics and Technology, Dolgoprudny, Institutsky per., 9, \\ 141700 Moscow Region, Russia \\ 4 Max-Planck-Institut für Radioastronomie, Auf dem Hügel 69, 53121 Bonn, Germany; \\ tsavolainen@mpifr-bonn.mpg.de \\ 5 Department of Physics and Astronomy, Purdue University, 525 Northwestern Avenue, \\ West Lafayette, IN 47907, USA; mlister@purdue.edu (M.L.L.); hodge2@perdue.edu (M.A.H.) \\ 6 Metsähovi Radio Observatory, Aalto University, Metsähovintie 114, FI-02540 Kylmälä, Finland \\ 7 Department of Electronics and Nanoengineering, Aalto University, PL 15500, FI-00076 Aalto, Finland \\ 8 Department of Astronomy, University of Michigan, 311 West Hall, 1085 S. University Avenue, \\ Ann Arbor, MI 48109, USA; mfa@umich.edu (M.F.A.); haller@umich.edu (H.D.A.) \\ * Correspondence: pushkarev.alexander@gmail.com \\ + This paper is an extended version of our paper published in Polarised Emission from Astrophysical Jets.
}

Received: 6 October 2017; Accepted: 27 November 2017; Published: 1 December 2017

\begin{abstract}
We used $15 \mathrm{GHz}$ multi-epoch Very Long Baseline Array (VLBA) polarization sensitive observations of 484 sources within a time interval 1996-2016 from the MOJAVE program, and also from the NRAO data archive. We have analyzed the linear polarization characteristics of the compact core features and regions downstream, and their changes along and across the parsec-scale active galactic nuclei (AGN) jets. We detected a significant increase of fractional polarization with distance from the radio core along the jet as well as towards the jet edges. Compared to quasars, BL Lacs have a higher degree of polarization and exhibit more stable electric vector position angles (EVPAs) in their core features and a better alignment of the EVPAs with the local jet direction. The latter is accompanied by a higher degree of linear polarization, suggesting that compact bright jet features might be strong transverse shocks, which enhance magnetic field regularity by compression.
\end{abstract}

Keywords: active galactic nuclei; relativistic jets; linear polarization; radio interferometry

\section{Introduction}

Despite substantial progress in the understanding of the phenomenon of the jets of active galactic nuclei (AGN) achieved over the last several decades with the method of very long baseline interferometry (which probes jets from sub- to several hundred parsec scales) the question of how jets are launched, confined and collimated remains an active area of research. The dominant acceleration mechanisms, which could be steady or impulsive in nature, are still unknown. All these questions are expected to be tightly connected with the key agent of jet dynamics, the magnetic (B) field. Construction of the Very Long Baseline Array (VLBA) in 1994 has allowed polarimetric observations of large samples of AGN jets on a regular basis facilitating detailed studies of the polarization characteristics of jet synchrotron radio emission.

VLBA polarization maps provide crucial information on the configuration of the magnetic field associated with an outflow, its regularity and orientation with respect to the local jet direction. In this 
publication, we analyze the polarization properties of a large sample of AGN jets mainly observed within the MOJAVE program [1] with the VLBA at $15 \mathrm{GHz}$.

\section{Observational Data and the Sample}

The data consist of 5410 polarization sensitive VLBA observations of 484 AGNs at 338 individual epochs between 1996 January 19 and 2016 December 26. The sources are drawn from a number of samples: the complete flux density-limited MOJAVE 1.5 sample [2], the joint gamma-ray and radio-selected sample 1FM [3], the VLBA $2 \mathrm{~cm}$ survey [4], the MOJAVE low-luminosity sample [5], the 3-rd EGRET gamma-ray catalog [6], and the 3FGL Fermi LAT gamma-ray catalog [7]. Most of the sources $(80 \%)$ have been detected at high energies by the Fermi LAT instrument [7]. All targets were bright enough ( $\gtrsim 50 \mathrm{mJy}$ ) at $15 \mathrm{GHz}$ for direct fringe detection on short integration times. We reduced the data in AIPS software package using standard techniques and performed imaging in Difmap [8].

The overwhelming majority $(88 \%)$ of the VLBA observations were done within the MOJAVE program [1], while the rest were obtained from the NRAO archive to increase the number of epochs for certain sources. The source cadence in the MOJAVE program is individually determined by the proper motion of jet knots and varies from about a month to two years. The median number of VLBA observation epochs per source is seven, although 54 sources have more than 20 epochs, with a maximum of 133 epochs for BL Lac. Since the beginning of the MOJAVE program in 2002, the noise level of the Stokes $Q$ and $U$ maps improved by a factor of $\sim 3$ and currently reaches a typical value of $\sim 0.1 \mathrm{mJy}^{\text {beam }}{ }^{-1}$, with a bit rate of $2 \mathrm{Gbps}$ and 2-bit sampling. The corresponding linear polarization and total intensity maps with a more detailed description of image characteristics are available in [9].

The sample is strongly dominated by flat-spectrum radio quasars $(71 \%)$, with a significant fraction of BL Lacertae objects $(20 \%)$, and a small fraction of radio galaxies $(7 \%)$. The rest of the sample $(2 \%)$ is comprised of optically unidentified sources. The redshifts are currently known for 443 objects $(91 \%)$, ranging from 0.00436 for the galaxy M87 to 3.636 for the BL Lac object 1549+089 and corresponding to scale factors ranging from 0.01 to $7.35 \mathrm{pc} \mathrm{mas}^{-1}$, respectively. Taking into account that a typical angular resolution of the VLBA observations at $15 \mathrm{GHz}$ is of the order of 1 mas, path lengths along the constructed ridgelines range from 1 to 57 mas and viewing angles are of the order of few degrees for blazars and may reach up to few tens of degrees for radio galaxies [10]. This implies that we probe absolute linear scales from sub- to hectoparsecs of the collimated AGN outflows.

\section{Results}

For the purposes of our analysis we produced maps of polarized intensity $P=\left(Q^{2}+U^{2}\right)^{1 / 2}$, electric vector position angle $\chi=0.5 \operatorname{atan}(U / Q)$, and fractional polarization $m=P / I$, where $I$ is the total intensity. Since the noise in a $P$ image is non-uniform and follows a Ricean distribution, we adopted a conservative approach of estimating the detection limit based on a level at which spurious $P$ signals appear in blank sky region of each map. Typically, this corresponds roughly to a $3 \sigma$ level, but in about $16 \%$ of the observations it exceeds 5 times $\sigma$ due to a high peak in a total intensity map or high instrumental polarization.

We extracted fractional linear polarization values from the constructed $m$-maps at the positions of total intensity components that were derived from structure model fitting performed in Difmap. If the polarization intensity of a component was lower than the noise level, we calculated a corresponding upper limit. In Figure 1 we plot the degree of polarization of the jet components of all sources separated by optical classification at all available epochs as a function of distance to the VLBA core. It shows a tendency for fractional polarization to increase with core separation implying that the magnetic field becomes more regular down the jet. Polarization of radio galaxies is weaker, especially for jet components within a few milliarcseconds from the core, with a significantly larger fraction of upper limits (53\%) compared to quasars $(23 \%)$ and BL Lacs $(22 \%)$. 

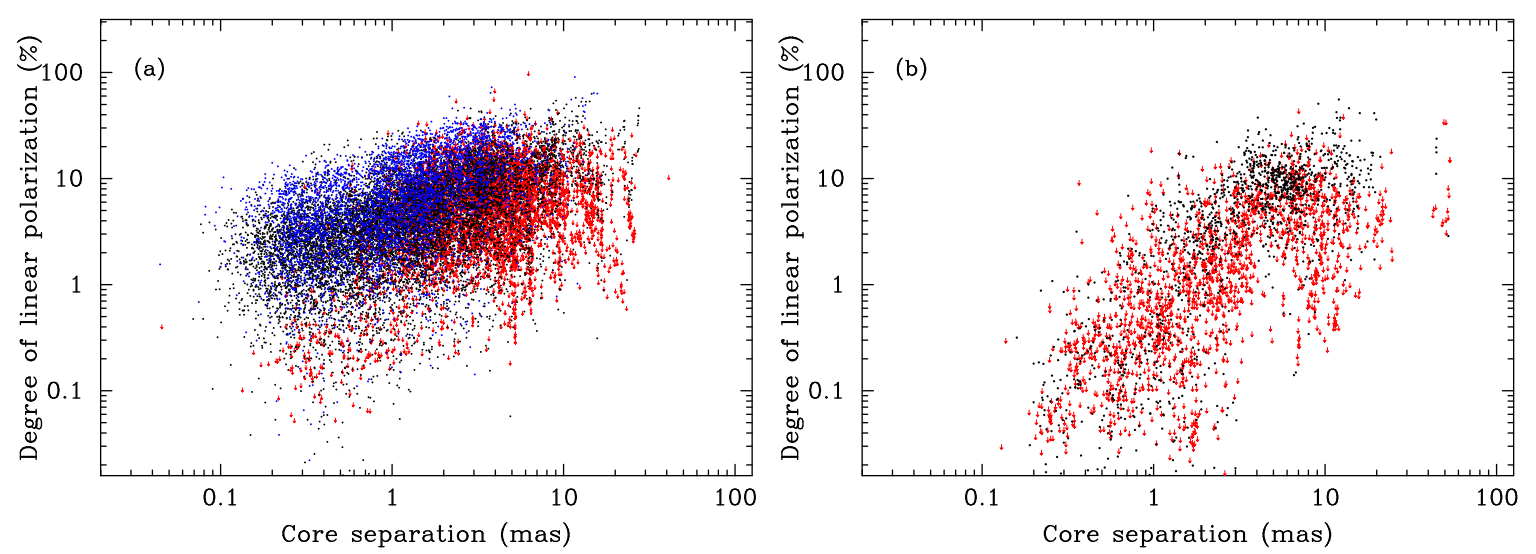

Figure 1. (a) Fractional polarization at positions of components in total intensity with distance from the $15 \mathrm{GHz}$ core for quasars (black) and BL Lacertae objects (blue). Dots show measurements, while red arrows represent upper limits. (b) Same for radio galaxies.

Core features typically have the highest levels of polarized flux density in the jet but lower fractional linear polarization. The cores, being partially optically thick, have typical degree of polarization on levels of a few per cent in quasars and BL Lacs, while the cores of radio galaxies are weakly polarized, with $m_{\text {core }}<0.5 \%$ (Figure 2). Low- and intermediate SED peak BL Lacs have the most highly polarized cores. In only 30 out of 5410 total epochs $m_{\text {core }}>10 \%$. Both core and jet components of BL Lacs, on average, are more polarized than those of quasars.
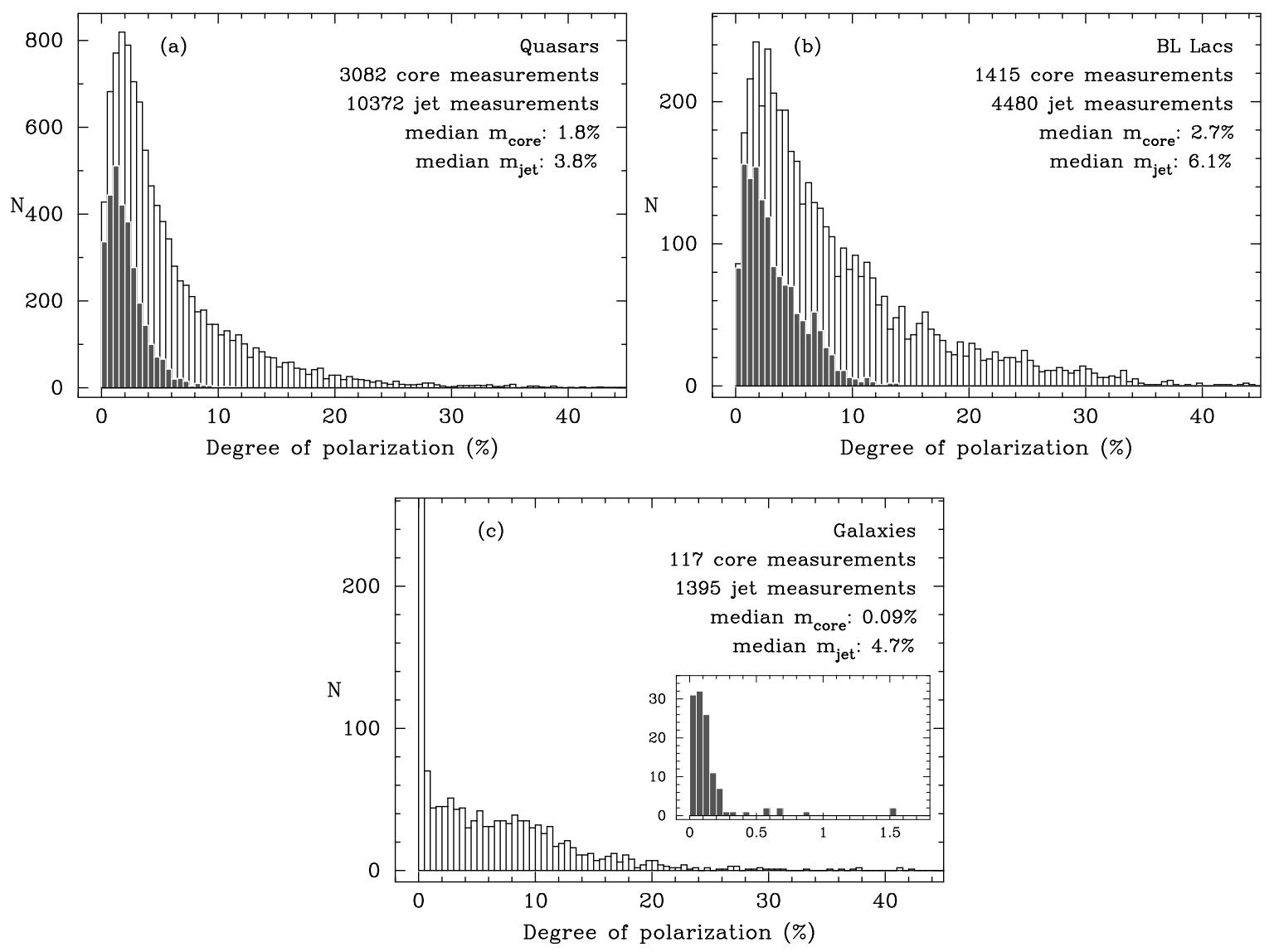

Figure 2. (a) Histograms of fractional polarization of core (filled gray bins) and jet components (empty bins) for quasars, (b) for BL Lacs, (c) for radio galaxies. 
We have also found that roughly $40 \%$ of the AGN jet cores show a tendency for a preferred EVPA direction over time. BL Lac cores have more stable EVPAs than those of quasars and show a tendency to be aligned with the inner parsec-scale jet. Similar behaviour is detected for the EVPA of jet components of BL Lacertae objects, which tend to be aligned with the local jet direction. In contrast, quasars and radio galaxies do not show such a trend. This confirms the findings of earlier studies, e.g., [11,12]. In many BL Lacs, high degrees of polarization are associated with low deviations of EVPAs from the jet direction (Figure 3).
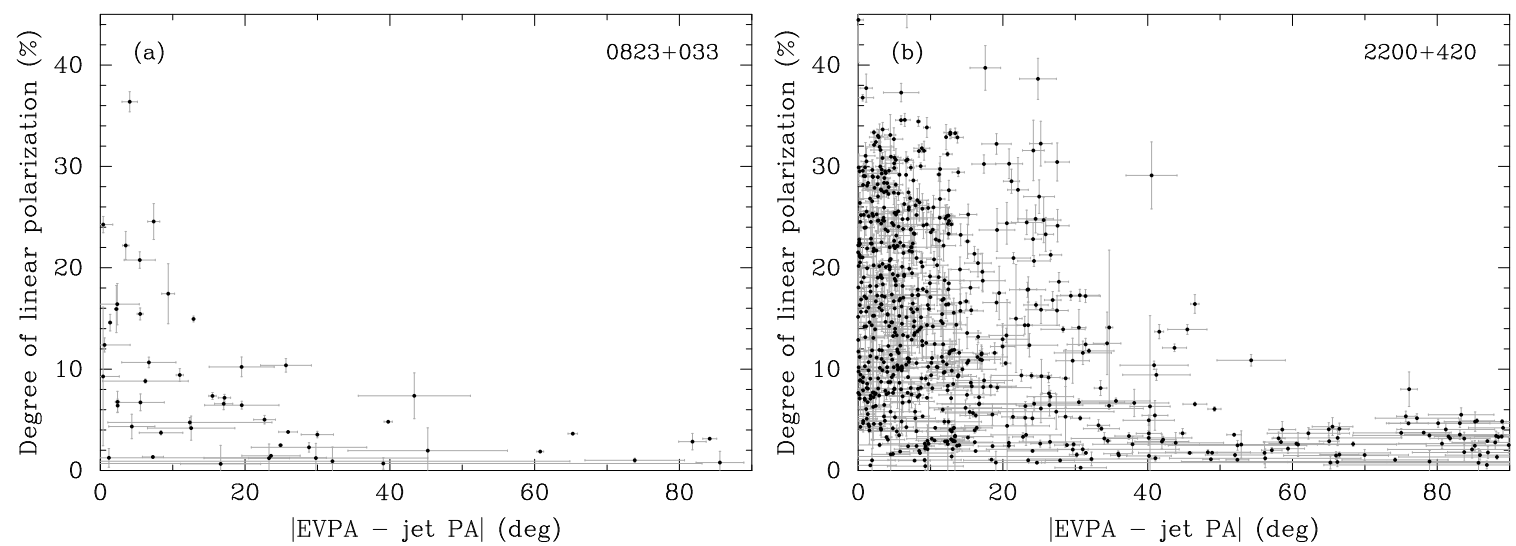

Figure 3. (a) Negative correlation between degree of polarization of total intensity components and absolute deviation of the electric vector position angles (EVPA) from the local jet direction for BL Lac object $0823+033$. (b) Same for BL Lac.

Fractional polarization maps taken within our program, and also in individual source studies, e.g., [13], often manifest clear increase of $m$-values towards the jet edges. To analyze changes in degree of polarization across the jet, we constructed jet ridgelines in total intensity following the procedure described in [10], and made $m$-slices transverse to the local jet direction. In Figure 4 we present examples of two sources, the radio galaxy $0430+052$ and quasar $1150+812$, that reveal rich polarization structure at $15 \mathrm{GHz}$ and have many epochs of observations. The sources show a characteristic V-shaped profile in the $m$-cuts (where the $x$-axis is a measure of offset from the ridgeline of the jet in units of restoring beams). Closer to the jet edges, the degree of polarization increases up to a few tens of per cent. Many other sources show similar tendency suggesting that this effect is quite common.
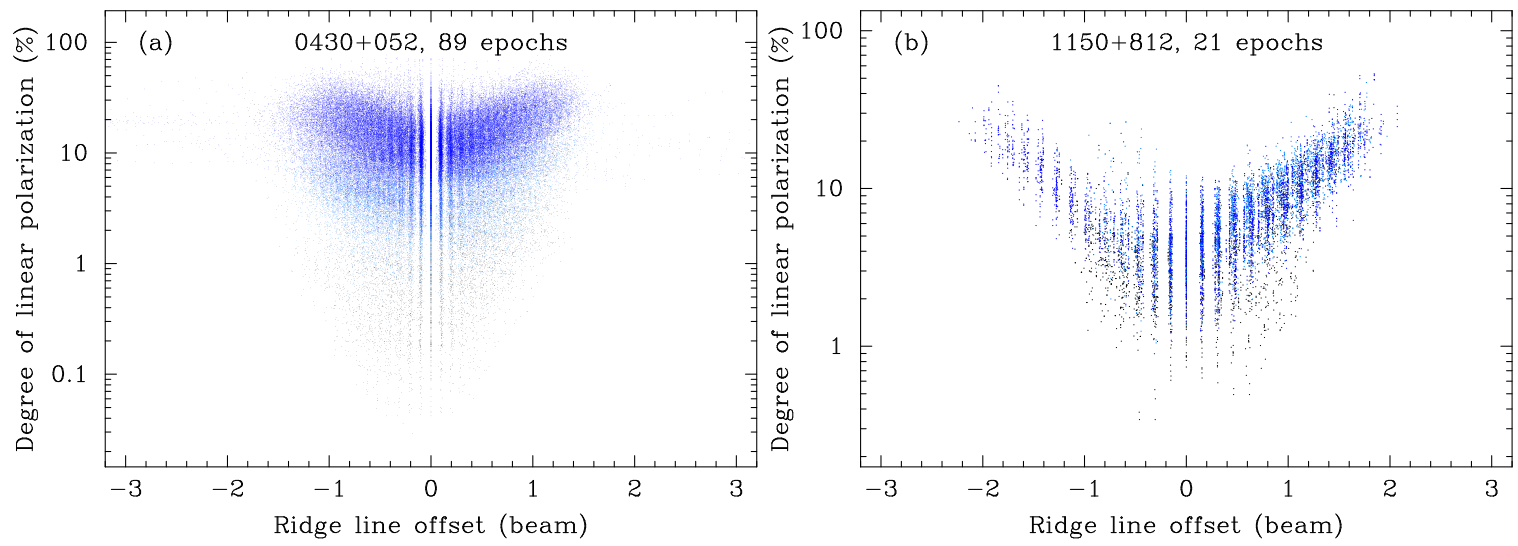

Figure 4. (a) Fractional polarization from slices transverse to the local jet direction as a function of offset from total intensity ridgeline for the radio galaxy 3C $120(0430+052)$. Black, cyan and blue dots show measurements at distances $r<1$ mas, $1<r<3$ mas, and $r>3$ mas from the $15 \mathrm{GHz}$ core measured along ridgeline, respectively. (b) Same for the quasar $1151+812$. 


\section{Discussion}

Since a jet expands with distance, magnetic field decreases as $B \propto r^{-b}$ ( $b=1$ for a pure toroidal field, $b=2$ for a pure axial field) but becomes more regular, as is evident from the observed increase in fractional polarization. One possible explanation of this trend is that magnetohydrodinamic turbulence is expected to decrease with distance from relativistic shocks and also down the jet [14]. The observed increase of the degree of polarization towards the jet edges is likely due to the superposition of synchrotron emission from regions with different magnetic field orientations resulting in depolarization, which is more efficient closer to the jet axis and weaker to the edges, where the emission layer is thinner.

The VLBA core features have a degree of polarization within $10 \%$ implying that synchrotron radiation from these regions is optically thick, while it is optically thin in the jet as $m_{\text {jet }}$ often exceeds this limit. In BL Lacs, the observed increase in alignment of EVPA with jet direction (perpendicular B-field) accompanied by a higher fractional polarization suggests that the bright jet components might be shock fronts that enhance magnetic field orderliness by compression. Alternatively, it may be interpreted in terms of a toroidal component of a large-scale helical B-field associated with the jet, though this scenario faces difficulties explaining inverse dependence between $\mid$ EVPA - jet PA $\mid$ and $m$ detected in BL Lacs.

Acknowledgments: The MOJAVE project was supported by NASA-Fermi grants NNX08AV67G, NNX12A087G, and NNX15AU76G. MFA was supported in part by NASA-Fermi GI grants NNX09AU16G, NNX10AP16G, NNX11AO13G, NNX13AP18G and NSF grant AST-0607523. This research has made use of data from the MOJAVE database that is maintained by the MOJAVE team [1]. This work made use of the Swinburne University of Technology software correlator [15], developed as part of the Australian Major National Research Facilities Programme and operated under licence. YYK and ABP are partly supported by the Russian Foundation for Basic Research (project 17-02-00197), the government of the Russian Federation (agreement 05.Y09.21.0018), and the Alexander von Humboldt Foundation. TS was supported by the Academy of Finland projects 274477 and 284495. The National Radio Astronomy Observatory is a facility of the National Science Foundation operated under cooperative agreement by Associated Universities, Inc.

Author Contributions: M.L.L., Y.Y.K., A.B.P. and T.S. reduced and analyzed the data. M.F.A. and H.D.A. obtained simultaneous single dish polarization observations for calibration of the VLBA measurements. M.A.H. calibrated EVPAs for a number of experiments.

Conflicts of Interest: The authors declare no conflict of interest.

\section{Abbreviations}

The following abbreviations are used in this manuscript:

AGN Active Galactic Nucleus

AIPS Astronomical Image Processing System

EVPA Electric Vector Position Angle

MOJAVE Monitoring Of Jets in Active galactic nuclei with VLBA Experiments

NRAO National Radio Astronomy Observatory

SED Spectral Energy Distribution

VLBA Very Long Baseline Array

\section{References}

1. Lister, M.L.; Cohen, M.H.; Homan, D.C.; Kadler, M.; Kellermann, K.I.; Kovalev, Y.Y.; Ros, E.; Savolainen, T.; Zensus, J.A. MOJAVE: Monitoring of Jets in Active Galactic Nuclei with VLBA Experiments. VI. Kinematics Analysis of a Complete Sample of Blazar Jets. Astron. J. 2009, 138, 1874-1892.

2. Lister, M.L.; Aller, M.F.; Aller, H.D.; Hovatta, T.; Max-Moerbeck, W.; Readhead, A.C.S.; Richards, J.L.; Ros, E. Why Have Many of the Brightest Radio-loud Blazars Not Been Detected in Gamma-Rays by Fermi? Astrophys. J. Lett. 2015, 810, doi:10.1088/2041-8205/810/1/L9. 
3. Lister, M.L.; Aller, M.; Aller, H.; Hovatta, T.; Kellermann, K.I.; Kovalev, Y.Y.; Meyer, E.T.; Pushkarev, A.B.; Ros, E.; Ackermann, M.; et al. $\gamma$-ray and Parsec-Scale Jet Properties of a Complete Sample of Blazars from the MOJAVE Program. Astrophys. J. Lett. 2011, 742, doi:10.1088/0004-637X/742/1/27.

4. Kellermann, K.I.; Vermeulen, R.C.; Zensus, J.A.; Cohen, M.H. Sub-Milliarcsecond Imaging of Quasars and Active Galactic Nuclei. Astron. J. 1998, 115, 1295-1318.

5. Lister, M.L.; Aller, M.F.; Aller, H.D.; Homan, D.C.; Kellermann, K.I.; Kovalev, Y.Y.; Pushkarev, A.B.; Richards, J. MOJAVE. X. Parsec-scale Jet Orientation Variations and Superluminal Motion in Active Galactic Nuclei. Astron. J. 2013, 146, doi:10.1088/0004-6256/146/5/120.

6. Hartman, R.C. The Third EGRET Catalog of High-Energy Gamma-Ray Sources. Astrophys. J. Suppl. Ser. 1999, 123, 79-202.

7. Acero, F.; Ackermann, M.; Ajello, M.; Albert, A.; Atwood, W.B.; Axelsson, M.; Baldini, L.; Ballet, J.; Barbiellini, G.; Bastieri, D.; et al. Fermi Large Area Telescope Third Source Catalog. Astrophys. J. Suppl. Ser. 2015, 218, doi:10.1088/0067-0049/218/2/23.

8. Shepherd, M.C. Difmap: An Interactive Program for Synthesis Imaging. In Astronomical Data Analysis Software and Systems VI; A.S.P. Conference Series; Hunt, G.; Payne, H.E.; Eds.; Astronomical Society of the Pacific: San Francisco, CA, USA, 1997; Volume 125, p. 77.

9. Lister, M.L.; Aller, M.F.; Aller, H.D.; Hodge, M.A.; Homan, D.C.; Kovalev, Y.Y.; Pushkarev, A.B.; Savolainen, T. MOJAVE: XV. VLBA $15 \mathrm{GHz}$ Total Intensity and Polarization Maps of 437 Parsec-Scale AGN Jets from 1996-2017. Astrophys. J. Suppl. Ser. 2017, in press.

10. Pushkarev, A.B.; Kovalev, Y.Y.; Lister, M.L.; Savolainen, T. MOJAVE-XIV. Shapes and opening angles of AGN jets. Mon. Not. R. Astron. Soc. 2017, 468, 4992-5003.

11. Gabuzda, D.C.; Pushkarev, A.B.; Cawthorne, T.V. Analysis of $\lambda=6 \mathrm{~cm}$ VLBI polarization observations of a complete sample of northern BL Lacertae objects. Mon. Not. R. Astron. Soc. 2000, 319, 1109-1124.

12. Lister, M. L.; Homan, D.C. MOJAVE: Monitoring of Jets in Active Galactic Nuclei with VLBA Experiments. I. First-Epoch 15 GHz Linear Polarization Images. Astron. J. 2005, 130, 1389-1417.

13. Pushkarev, A.B.; Gabuzda, D.C.; Vetukhnovskaya, Y.N.; Yakimov, V.E. Spine-sheath polarization structures in four active galactic nuclei jets. Mon. Not. R. Astron. Soc. 2005, 356, 859-871.

14. Böttcher, M. Spectral and Polarization Signatures of Relativistic Shocks in Blazars. Galaxies 2016, 4, doi:10.3390/galaxies4030022.

15. Deller, A.T.; Brisken, W.F.; Phillips, C.J.; Morgan, J.; Alef, W.; Cappallo, R.; Middelberg, E.; Romney, J.; Rottmann, H.; Tingay, S.J.; et al. DiFX-2: A More Flexible, Efficient, Robust, and Powerful Software Correlator. Publ. Astron. Soc. Pac. 2011, 123, 275-287.

(C) 2017 by the authors. Licensee MDPI, Basel, Switzerland. This article is an open access article distributed under the terms and conditions of the Creative Commons Attribution (CC BY) license (http://creativecommons.org/licenses/by/4.0/). 